\title{
Retinal fundus imaging with a plenoptic sensor
}

Brice Thurin, Edward Bloch, Sotiris Nousias, Sebastien Ourselin, Pearse Keane, et al.

Brice Thurin, Edward Bloch, Sotiris Nousias, Sebastien Ourselin, Pearse Keane, Christos Bergeles, "Retinal fundus imaging with a plenoptic sensor," Proc. SPIE 10474, Ophthalmic Technologies XXVIII, 1047429 (19 February 2018); doi: $10.1117 / 12.2286448$

SPIE. Event: SPIE BiOS, 2018, San Francisco, California, United States 


\title{
Retinal fundus imaging with a plenoptic sensor
}

\author{
Brice Thurin ${ }^{\mathrm{a}, \mathrm{b}}$, Edward Bloch ${ }^{\mathrm{a}, \mathrm{c}}$, Sotiris Nousias ${ }^{\mathrm{a}, \mathrm{b}}$, Sebastien Ourselin ${ }^{\mathrm{a}, \mathrm{b}}$, Pearse A. Keane ${ }^{\mathrm{c}}$, \\ and Christos Bergeles ${ }^{\mathrm{a}, \mathrm{b}}$ \\ ${ }^{a}$ Wellcome/EPSRC Centre for Interventional and Surgical Sciences, University College \\ London, London, UK \\ ${ }^{\mathrm{b}}$ Translational Imaging Group, Centre for Medical Image Computing, Department of Medical \\ Physics and Biomedical Engineering, University College London, London, UK \\ 'NIHR Biomedical Research Centre, Moorfields Eye Hospital, NHS Foundation Trust, London, \\ UK
}

\begin{abstract}
Vitreoretinal surgery is moving towards 3D visualization of the surgical field. This require acquisition system capable of recording such 3D information. We propose a proof of concept imaging system based on a light-field camera where an array of micro-lenses is placed in front of a conventional sensor. With a single snapshot, a stack of images focused at different depth are produced on the fly, which provides enhanced depth perception for the surgeon.

Difficulty in depth localization of features and frequent focus-change during surgery are making current vitreoretinal heads-up surgical imaging systems cumbersome to use. To improve the depth perception and eliminate the need to manually refocus on the instruments during the surgery, we designed and implemented a proof-of-concept ophthalmoscope equipped with a commercial light-field camera. The sensor of our camera is composed of an array of micro-lenses which are projecting an array of overlapped micro-images. We show that with a single light-field snapshot we can digitally refocus between the retina and a tool located in front of the retina or display an extended depth-of-field image where everything is in focus.

The design and system performances of the plenoptic fundus camera are detailed. We will conclude by showing in vivo data recorded with our device.
\end{abstract}

Keywords: plenoptic fundus camera, light-field ophthalmoscope, vitreoretinal surgery, image guided surgery, Three dimensional ophthalmic imaging

\section{INTRODUCTION}

In heads-up vitreoretinal surgery,${ }^{1}$ the surgeon operates on the patient by looking at a display instead of looking through the eye piece of the surgical microscope. The advantages are better ergonomics, reduced illumination levels and 3D imaging available to the entire surgical team. The images are obtained via a surgical stereo microscope. In addition, real time digital video processing provides enhanced contrast, brightness and dynamic range. Current heads-up surgical imaging systems, however, suffer from a short depth of focus, especially at high magnification. This introduces difficulty in depth localization of features, and requires frequent focus change during surgery. ${ }^{2}$ As with conventional retinal imaging, these limitations could be improved by reducing the aperture of the imaging optics at the cost of dangerously reducing depth-perceiving cues, and potentially reaching phototoxic illumination levels.

Complementary or alternative technology such as the coupling of the surgical microscope with a real time optical coherence tomographer (OCT) has been proposed to facilitate the surgery. ${ }^{3}$ This additional imaging modality provide a depth resolution of the order of a few tenth of micrometers to the surgeons for precise instruments placement within a limited field of view of up to 10 by 10 millimeters. But the wide field 3D

Further author information: (Send correspondence to Brice Thurin or Christos Bergeles)

Brice Thurin: E-mail: b.thurin@ucl.ac.uk

Christos Bergeles: E-mail: c.bergeles@ucl.ac.uk

Ophthalmic Technologies XXVIII, edited by Fabrice Manns, Per G. Söderberg, Arthur Ho, Proc. of SPIE

Vol. 10474, $1047429 \cdot$ @ 2018 SPIE · CCC code: 1605-7422/18/\$18 - doi: 10.1117/12.2286448 
localization could still be improved, as the OCT surgical methods revealed previously unrecognized instrumenttissue interaction occurring during surgery.

In order to improve the depth perception and eliminate the need to manually refocus on the instruments retinal imaging, both for diagnostic examinations and during the surgery, it has been proposed that the surgical microscope be upgraded with a plenoptic sensor. ${ }^{4}$ Also known as a light-field camera, the plenoptic sensor is a standard electronic detector array with a matrix of micro-lenses in front of it. ${ }^{5-7}$ The micro-lenses project multiple viewpoint of a scene. The parallax between the micro-images allows $3 D$ reconstruction of the scene, and the computational refocusing of the image and video stream on-the-fly. ${ }^{8}$ Therefore, by incorporating the plenoptic sensor, $3 D$ in-focus video streams may be acquired intra-operatively.

Plenoptic ophthalmoscopy has been considered for eye examinations. ${ }^{9-11}$ A crude implementation has been proposed by Adam et al., ${ }^{12}$ the system built is used as a substitute for a human observer, only a small portion of the field is used for fundus imaging and the it does not exploit the full capabilities of light-field imaging. More recently a plenoptic sensor has been used to successfully characterize the topography of the healthy and diseased human iris in vivo. ${ }^{13}$

In this proceeding we present the design principle of a plenoptic fundus camera and an example of the implementation of such a system equipped with a commercial $3 D$ light-field camera. We show that with a single light-field snapshot we can digitally refocus between the retina and a tool located in front of the retina or display an extended depth of field image where everything is in focus.

\section{PLENOPTIC FUNDUS CAMERA DESIGN}

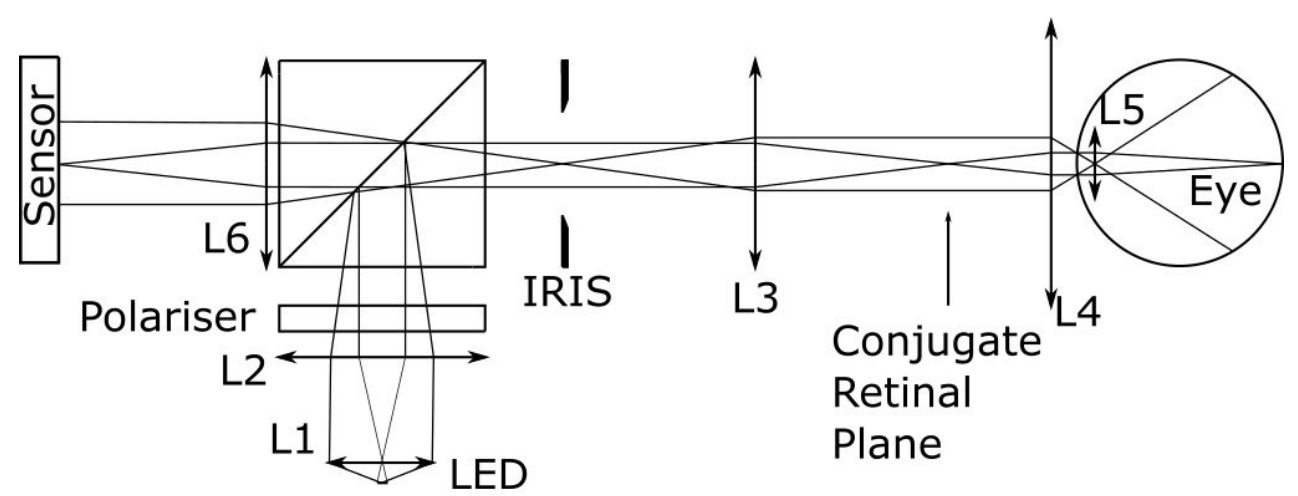

Figure 1. Optical layout of the fundus camera. A white LED illuminates the eye fundus through a polarizer and polarizing beamsplitter. The LED chip is conjugated with the eye pupil and an IRIS. While the condensing lens L1 is conjugated with the retinal plane.A primary image of the retinal is formed by a Digital Wide-Field lens L4. This image is relayed to the plenoptic sensor by L3 and L6 through the polarizing beamsplitter. The polarization helps reduce the corneal reflex.

The aim of building a fundus imaging system around a plenoptic sensor is to be able to recover depth information which is lost in conventional fundus camera. In order to do so, it is important to maximize the parallax and therefore image through the whole eye pupil diameter. Conventional fundus camera are limiting the imaging aperture to the central part of the eye pupil ${ }^{14}$ usually to a diameter smaller than $3 \mathrm{~mm}$. The imaging resolution is better for smaller pupil diameter due to ocular optical aberration. ${ }^{15}$ Another advantage is that the illumination path use the outer part of the pupil, therefore eliminating any potential spurious back reflexion from the cornea. Hence the design requirements for a plenoptic fundus camera differs from standard system as the entrance aperture of the imaging system is set by the physical size of the eye pupil.

The fundamental design principle for lightfield imaging is that the effective f-number of the micro-lenses array $\left(f_{\#}\right)$ and the main lens $\left(f_{\#}^{L}\right)$ are matched, ${ }^{5}$ this condition maximize the fill factor of the sensor while avoiding overlap between micro-images. By definition, $f_{\#}^{L}$ is the ratio of the effective focal length of the main 


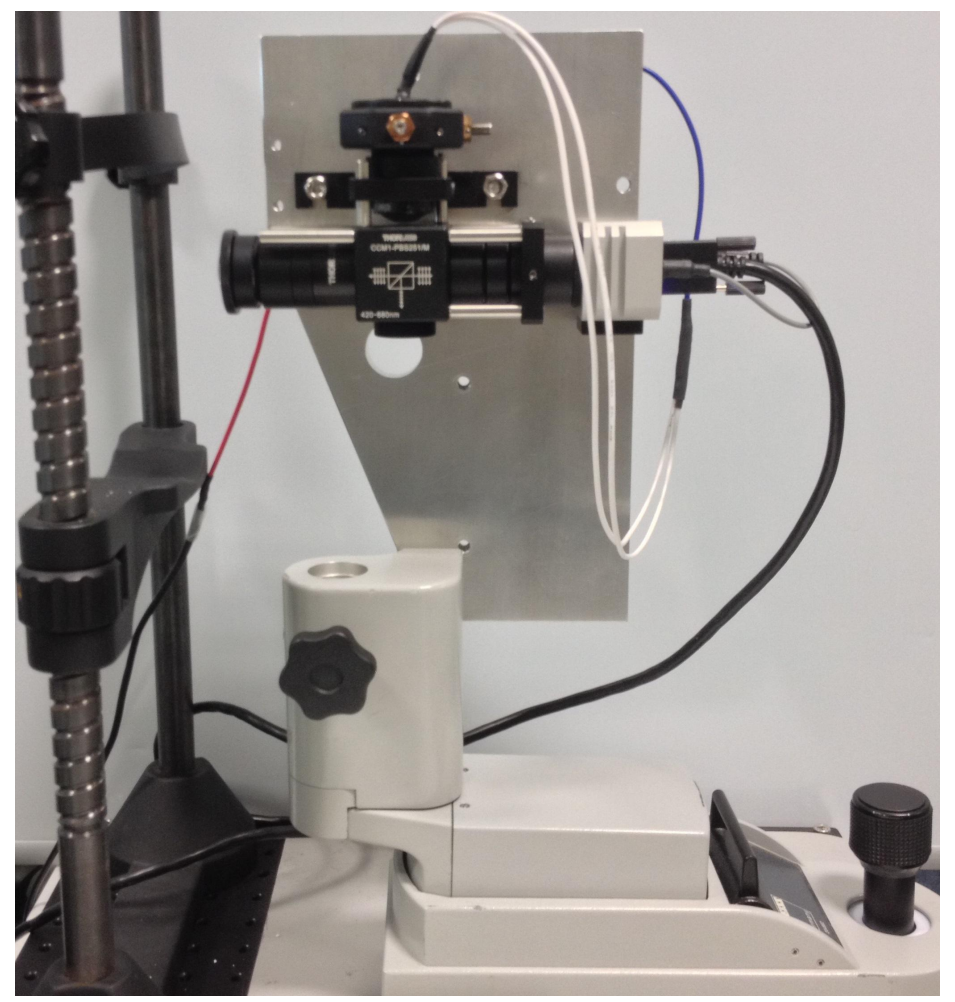

Figure 2. Lightfield ophthalmoscope prototype mounted on top of a slit lamp frame.

lens $\left(f^{L}\right)$ to the entrance pupil diameter which in our case is the diameter of the eye pupil $\left(d_{\text {eye }}\right)$ :

$$
f_{\#}=f_{\#}^{L}=\frac{f^{L}}{d_{\text {eye }}} .
$$

The field of the view of the system is set by the dimension of the sensor $\left(d_{s}\right)$ and $f^{L}$ such that

$$
\tan \left(f_{o v}\right)=\frac{d_{s}}{f^{L}}=\frac{d_{s}}{f_{\#} \times d_{\text {eye }}} .
$$

Hence if the f-number of the plenoptic sensor is predefined, the focal length of the main lens is set by the eye pupil diameter and the field of view will only depend on the size of the sensor.

The 3D lightfield sensor used in this study is a $\mathrm{R} 8$ from Raytrix $G m b H$. This is a multifocus plenoptic sensor, ${ }^{16,17}$ where there are three sets of lenslet interlaced with different focal length. This configuration provides an extended depth of field compared with lens arrays composed of lenses having all the same focal length. For this sensor, the effective image side f-number of the micro-lenses is fixed with a value of $f_{\#}=2.8$. For the design of our camera we assumed a nominal iris diameter of $d_{\text {eye }}=7 \mathrm{~mm}$, this is in accordance with the value assumed in the latest international standards when considering dark adapted or dilated pupil diameter. ${ }^{18}$ Therefore, according to equation 1, the focal length of the main lens of our proof of concept system should be equal to $f^{L}=19.6 \mathrm{~mm}$. The nominal field of view of our system is limited by the size of the Raytrix sensor and the f-number of its microlens array, with a diagonal of $27^{\circ}$ (width $=24^{\circ}$, height $=13^{\circ}$ ). For comparisons, in the operative theater, the field of view available to the surgeons can be as small as $30^{\circ},{ }^{19}$ but some of the latest surgical microscope are offering a working field of view reaching $100^{\circ} .^{20}$

The optical layout of our proof of concept system is presented in figure 1 and a picture of the built prototype in figure 2 . The retina is illuminated with a white LED synchronized with the camera acquisition. A polarizing beamsplitter and a polarizer are used to limit the unwanted reflexion from the cornea, L3 and L4; the illumination 


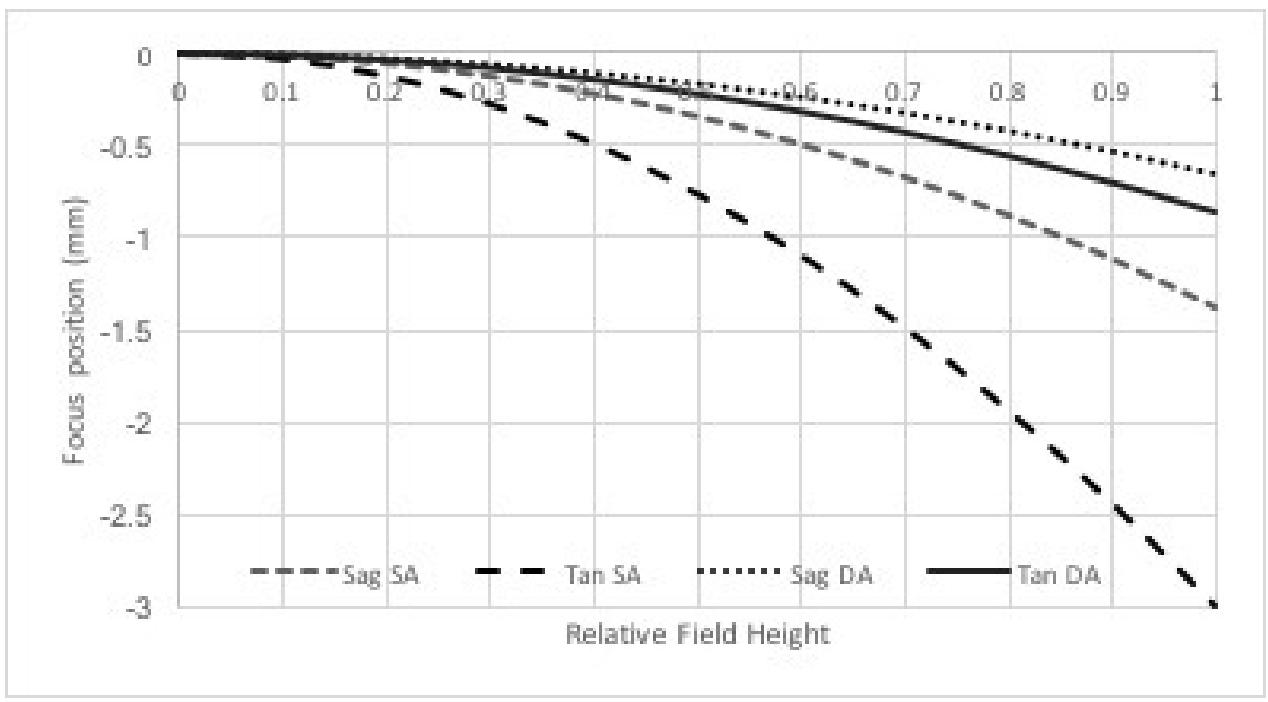

Figure 3. Field curve for the 4-f telecentric assembly for single achgromat (SA) and double achromat (DA) configurations.(Sag: sagital ray fan, Tan: tangential ray fan).

and imaging common path. As stated earlier commercial fundus camera use an annular ring conjugated with the pupil to illuminate the retina. The ring is located outside the $3 \mathrm{~mm}$ imaging entrance pupil, ${ }^{21}$ therefore avoiding the reduction in contrast of the retinal image due to the corneal reflex. Surgical microscopes do not suffer of this limitation as they are making use of chandelier illumination system. ${ }^{20} \mathrm{~A}$ long pass glass color filter with a cut-off at $495 \mathrm{~nm}$ filter out the blue emission peak of the LED.

A Digital Wide-Field non contact slit lamp lens from Volk (Part \# VDGTLWF) with a power of $\phi_{4}=90 D$ (L4) creates an aerial retinal image, that is then relayed on the sensor by a 4-f telecentric lens assembly (L3 and L6). The magnification of the telescope is given by

$$
M=\phi_{4} d_{\text {eye }} f_{\#} \sim 1.8
$$

in order to satisfy the f-number matching condition. For the design of our proof of concept system we are using off-the-shelf optical lenses with a diameter of up to $25.4 \mathrm{~mm}$. This limitation associated with the large field of view and $7 \mathrm{~mm}$ eye pupil diameter impose the use of lenses with focal length of $20 \mathrm{~mm}$ and $36 \mathrm{~mm}$ for L3 and L6 respectively. A single achromatic lens with these relatively short focal lengths exhibit a significant amount of field curvature and astigmatism. Instead to improve the imaging performances, L3 and L6 are splits into two achromatic doublets. As can be seen in figure 3, this significantly reduces astigmatism and field curvature.

The telecentric assembly (L3-L6) was optimized using the optical design software CodeV(Synopsys Inc). The optimization was done for the emmetropic conjugate plane configuration only. The listing for the as-built assembly is listed in table 1.

The post-capture processing capabilities of the lightfield sensor provides a mean of correcting for the field curvature and digitally refocus to planes located in front of the retina. Using the digital refocusing for field curvature correction should be avoided, such that most of the depth focus is used for practical application. The sensor used in this study provides a virtual depth refocusing ranging from 2 to 24 . The virtual depth $(v)$ is the ratio of the main lens image plane to lenslet plane distance $(a)$ over the sensor plane to lenslet plane distance $(B),{ }^{16}$

$$
v=\frac{a}{B}=\frac{a}{d \times f_{\#}}
$$

where $d$ is the diameter of one of the lenslet. $d$ is estimated to be $\sim 78 \mu \mathrm{m}$ from the sensor pixel size $(2.24 \mu \mathrm{m})$, the width resolution of the sensor (3840 pixels) and the approximate number of lenslet across the width of the sensor (110.5 lenslets). The depth of focus of the $\mathrm{R} 8$ sensor in the main lens image space is therefore given by 
Table 1. Lens listing as-built $4 \mathrm{f}$ telecentric lens assembly (L3-L6). Dimensions in (mm). L3-A, L3-B, L6-A and L6-B are cemented achromatic doublet (Thorlabs Inc) with respectively 35mm (AC254-035-A), 35mm (AC254-035-A), 75mm (AC254-075-A) and 60mm (AC254-060-A) focal length.

\begin{tabular}{ccccc}
\hline Surface & Element & Radius & Thickness & Glass \\
\hline 1 & L3-A & 102.1 & 2.0 & NSF6HT-Schott \\
2 & L3-A & 19.1 & 12.0 & NBAF10-Schott \\
3 & L3-A & -24.0 & 12.1 & \\
4 & L3-B & 102.1 & 2.0 & NSF6HT-Schott \\
5 & L3-B & 19.1 & 12.0 & NBAF10-Schott \\
6 & L3-B & -24.0 & 7.5 & \\
7 & Beamsplitter & & 8.5 & NBK7-Schott \\
Stop & Beamspitter & & 16.5 & NBK7-Schott \\
9 & Beamsplitter & & 9.5 & \\
10 & L6-A & 46.5 & 7.0 & NBK7-Schott \\
11 & L6-A & -33.9 & 2.5 & SF5-Schott \\
12 & L6-A & -95.5 & 24.0 & \\
13 & L6-B & 41.7 & 8.0 & EBAF11-Hikari \\
14 & L6-B & -25.9 & 2.5 & FD10-Hoya \\
15 & L6-B & -230.7 & 28.0 & \\
\hline
\end{tabular}

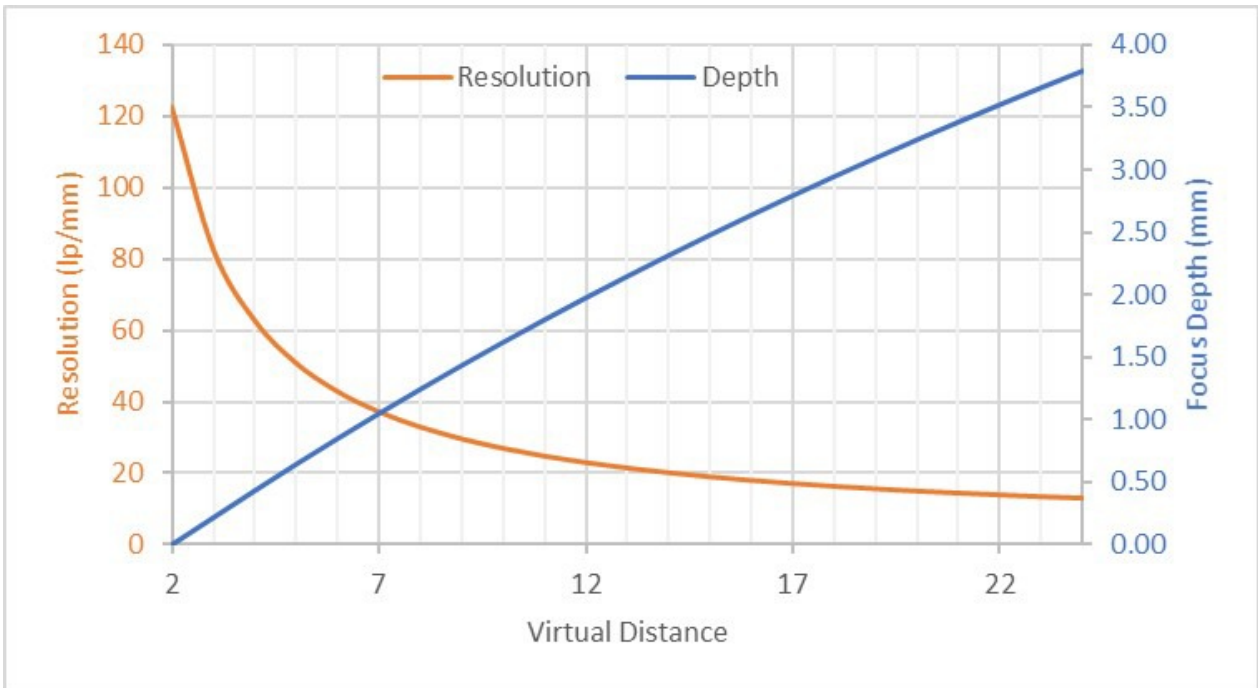

Figure 4. Focusing distance from the retina within the vitreous for different virtual depth and intra-ocular feature lateral resolution for different virtual distance. The resolution drop as the virtual distance increase.

the difference between $a_{\min }$ and $a_{\max }$ and is equal to $4.8 \mathrm{~mm}$. We simulated the refocusing plane distance from the retina into the vitreous against virtual depth (figure 4) for an eye with an effective focal length of $17 \mathrm{~mm}$ and taking into account the refractive index of the vitreous humor $(\sim 1.336)$.

We also estimated the theoretical lateral resolution of our system in retinal space for different refocusing plane. The virtual depth for the focused plenoptic camera is the inverse of the magnification of the lenslet array or in other word the effective pixel size of the sensor for each refocusing depth is equal to the product of the virtual depth of this plane by the physical pixel size of the image sensor. Hence as the virtual depth increase the lateral resolution is getting worse. Note that there is also a change in magnification for the main lens and ocular lens assembly between the different conjugate planes at different virtual depth. However the magnification of the lenslet across the depth of focus decrease by an order of magnitude, while the magnification of the rest 
of the optics increase by less than $40 \%$. For the resolution plot of figure 4 we took into account the overall magnification. This significant loss of resolution could be problematic in the context of an ophthalmoscope. In order to increase the focusing range of our system we would have to reduce the magnification of the main lens, at the same time this will reduce the field of view available for a given sensor size and require a short focal length lens. For a set eye pupil diameter, this will imply to reduce the micro-lenses f-number as defined by equation 1 . It is important to note that for this simulation we do not take into account for the resolution of the blurring effect due to the micro-images getting out of focus.

\section{TEST AND PERFORMANCES}

\subsection{Prototype Opththalmoscope Evaluations}

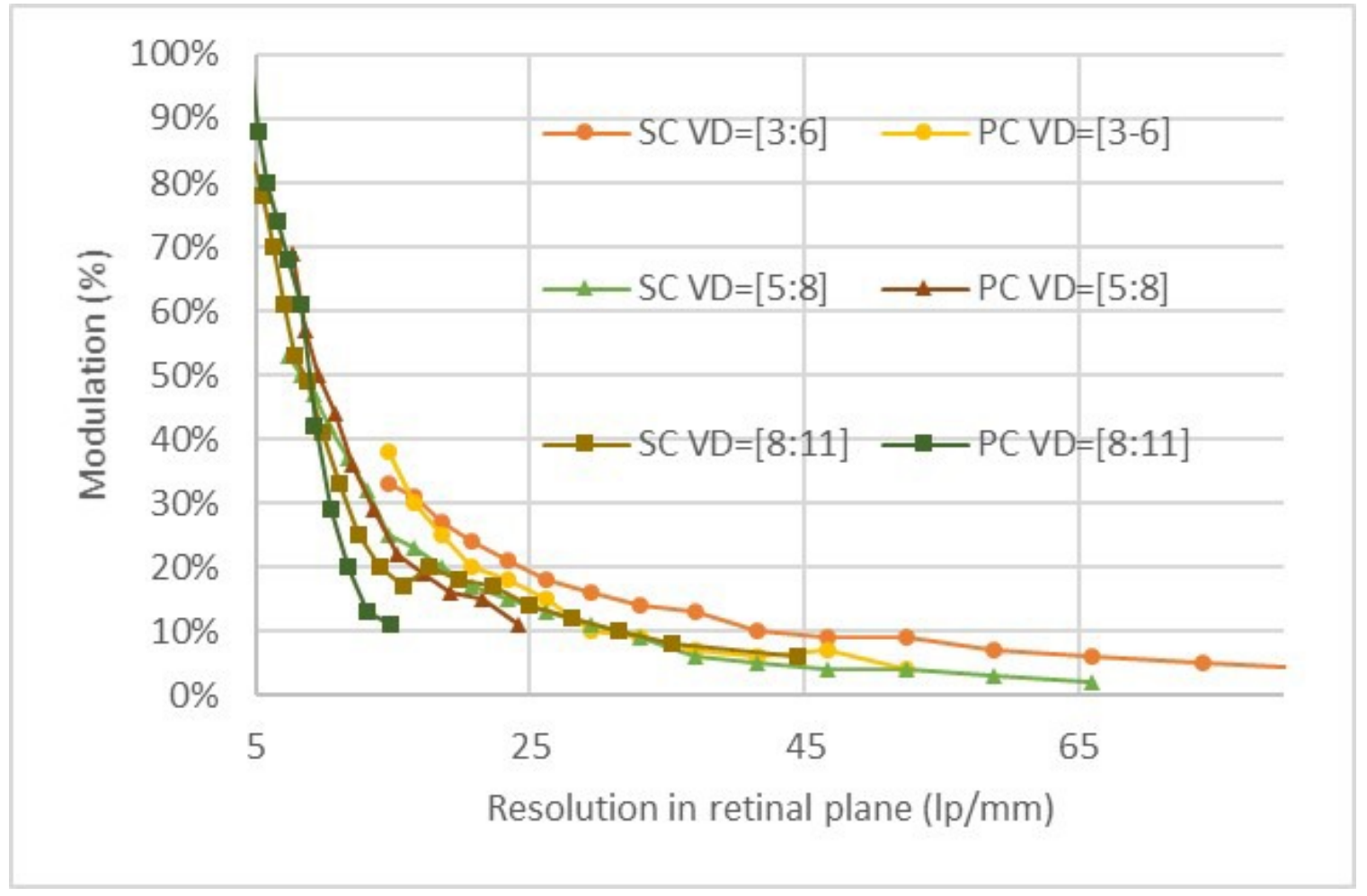

Figure 5. Modulation Transfer Function measurement for the prototype lightfield ophthalmoscope lens with the plenoptic camera (PC) and a standard Cmos camera (SC). Printed USAF 1951 targets were located at at $300 \mathrm{~mm}(\square), 500 \mathrm{~mm}(\triangle)$ and $1000 \mathrm{~mm}(\circ)$ from the fundus camera

The prototype' performance was measured by imaging printed 1951 USAF Resolution Targets that were placed between $300 \mathrm{~mm}$ and $1 \mathrm{~m}$ away from the system. First, to evaluate the optics independently, the plenoptic sensor (PC) was replaced with a standard CMOS camera (SC) (IDS, UI-3240CP-NIR-GL) with a pixel size of $5.3 \mu \mathrm{m}$ and the MTF was calculated. Subsequently, PC was introduced to the system, and the MTF was re-estimated. Figure 5 depicts the Modulation normalised at $0 \frac{\mathrm{lp}}{\mathrm{mm}}$ as a function of resolution of the different set of bars present on the chart for the target located at $300 \mathrm{~mm}(\square), 500 \mathrm{~mm}(\triangle)$ and $1000 \mathrm{~mm}(\circ)$, for PC and SC, respectively. The MTF curves obtained for SC exhibit a higher resolution than the one obtained with PC. For a virtual depth $v \approx 8$, SC could resolve features up to $45 \frac{\mathrm{lp}}{\mathrm{mm}}$, while $\mathrm{PC}$ could resolve $15 \frac{\mathrm{lp}}{\mathrm{mm}}$ features. This is to be compared with the value shown in Fig. 4 where we estimated the resolution to be $>20 \frac{\mathrm{lp}}{\mathrm{mm}}$ for $8<v<11$. In that simulation, the resolution in retinal space was estimated from the pixel size and the magnification of the micro-lens and lens combination. The blurring effect due to the defocused micro-lens images was not taken into account. This demonstrates that this omission may overestimate system performance. 


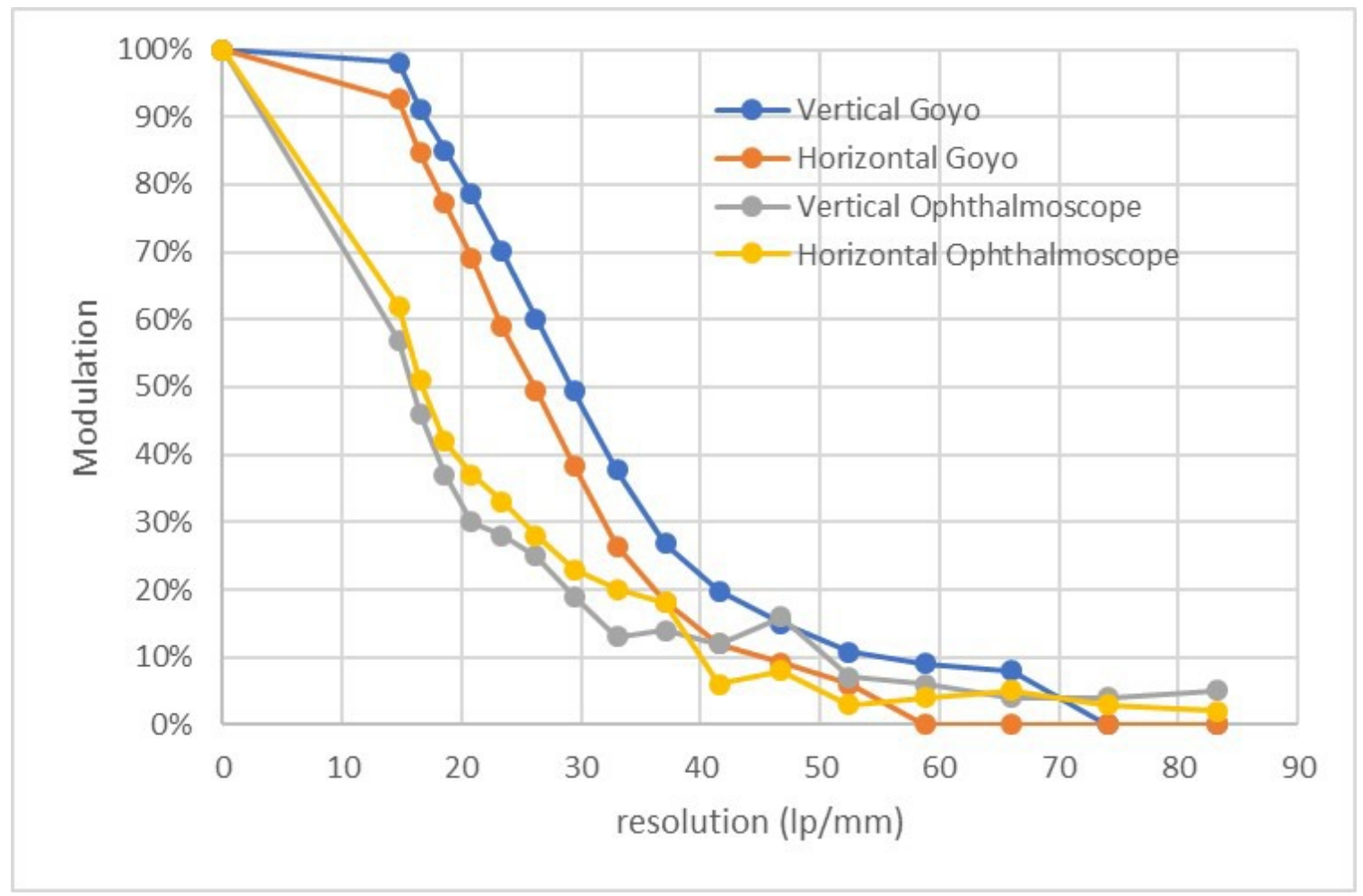

Figure 6. Modulation Transfer Function measurement comparison between a standard 20mm focal length camera lens manufactured by Goyo and the prototype ophthalmoscope lens. In both case the R8 lightfield camera was used and the lens focused such that best digital focus was obtained for a virtual distance of 4.0. Printed USAF 1951 targets were located at a distance of $1000 \mathrm{~mm}$ (o) from the lens.

Then we replaced the modulation transfer function of the plenoptic sensor assembled with a $20 \mathrm{~mm}$ camera lens from Goyo Optical (Model number: GM12HR42016MCN) with the 1951 USAF Resolution Targets $1 \mathrm{~m}$ away from the lens. The Goyo Lens is a well corrected camera lens rated for 1 inch 12 Mega-pixel sensor. The results were compared with the data obtained with those of the plenoptics sensor mounted on the ophthalmoscope. Figure 6 shows the modulation transfer curve obtained. It can be seen that below $40 \frac{l p}{\mathrm{~mm}}$ the Goyo lens significantly outperform our ophthalmoscope. For higher spatial frequency the modulation transfer function is limited by the plenoptic sensor.

\subsection{Artificial Eye Imaging}

Prior to conduct the in vivo experiment, we did some experiment with a Model Practice Eye (GWB International). The model eye has a pupil diameter equal to $8 \mathrm{~mm}$ and focal length of about $35 \mathrm{~mm}$. The model eye is twice the typical value of the human of $17 \mathrm{~mm}$. The focusing range of the model eye is therefore larger around $10 \mathrm{~mm}$.

We introduced a needle through the sclera of the model eye in order to simulate a surgical tool. Figure 7 shows a plenoptic images collected for the model with the needle inserted. The needle which is closer to the eye lens is imaged by more micro-lenses than the painted optic disc further away. Also it can noticed that the micro-images are well separated and are not overlapping each other. Figure 8 and 9 shows image refocus images on the retina and the needle respectively generated from the plenotpic image of figure 7 .

The RxLive software provided with the R8 provides a mean to produce a total focus image (fig. 10) and to generate a 3D depth map (fig. 11). However to produce meaningful result the object being imaged as to exhibit significant across the whole field of view. In addition the image has to be of high enough resolution without being too noisy. 


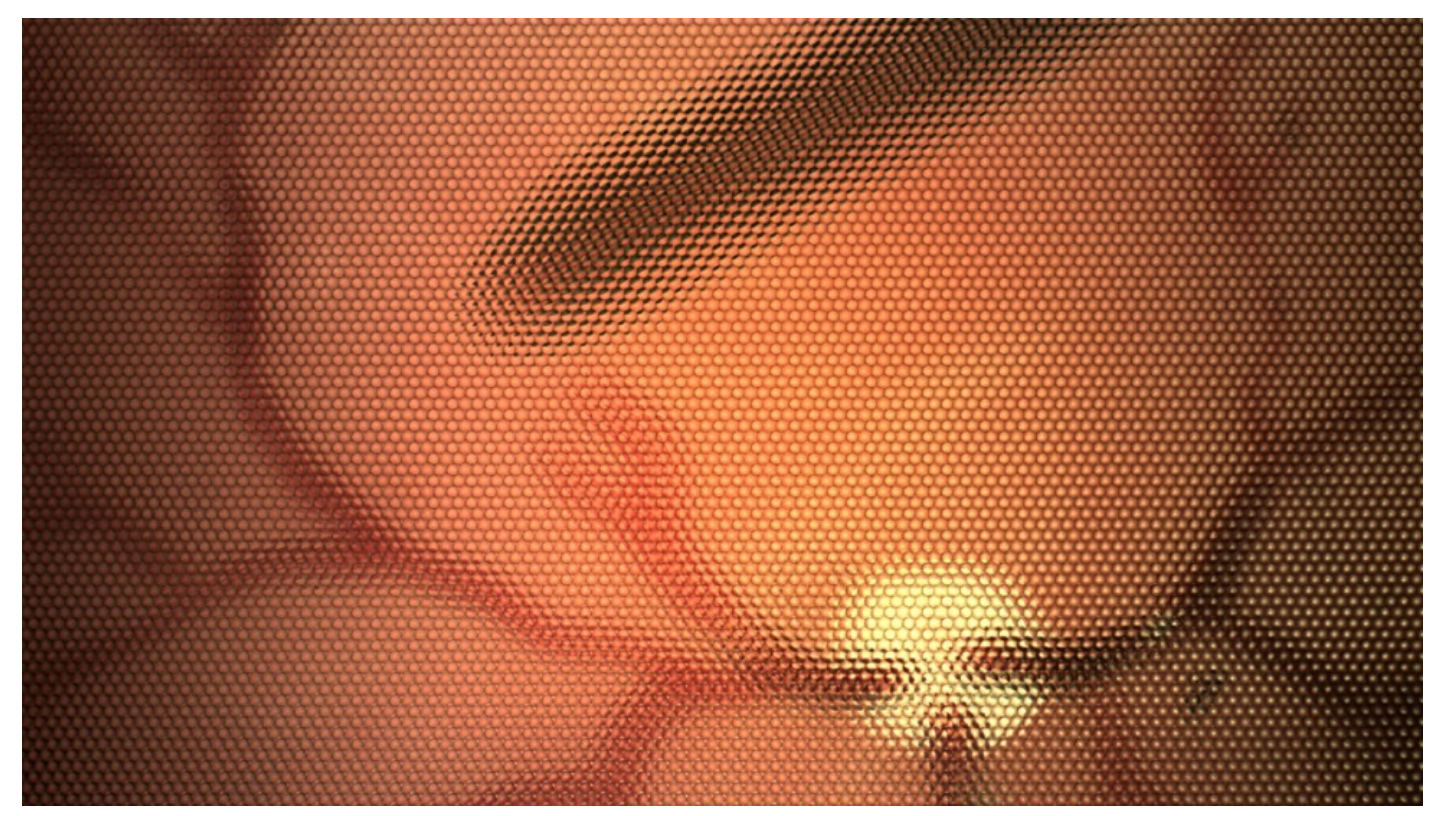

Figure 7. Plenotpic image of a Model Practice Eye from GWB International with a needle inserted through the sclera. This image was collected with the prototype ophthalmoscope describe in this proceeding.

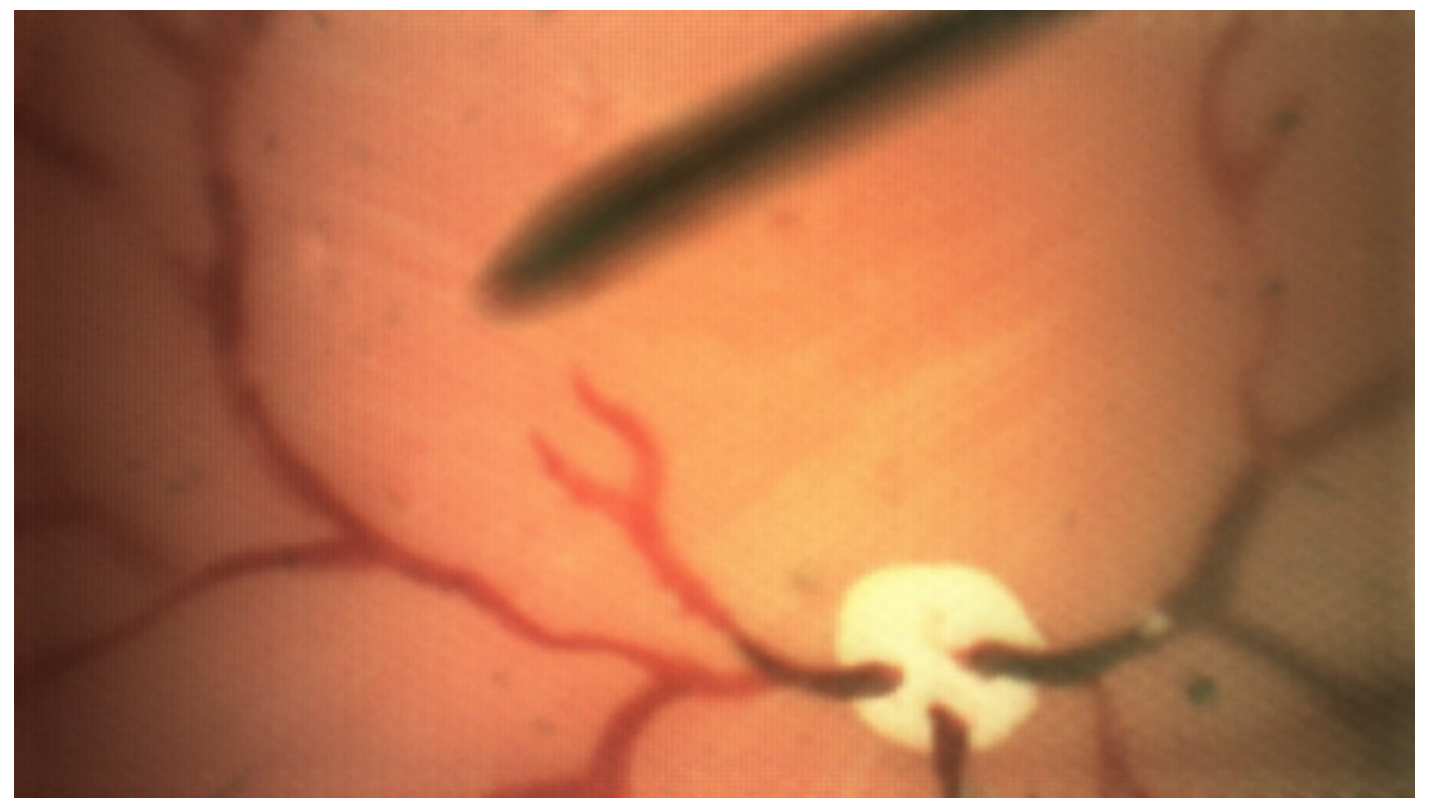

Figure 8. Digitally focused image on the retina using Raytrix RxLive Software generated from fig. 7

\subsection{In Vivo Imaging}

The weighted retinal irradiance is set so that its value is $<220 \frac{\mu W}{\mathrm{~cm}^{2}}$, which is the limit for continuous illumination for group 1 fundus camera, i.e. ophthalmic instrument for which no potential light hazard exists. ${ }^{18}$ We obtain ethical approval from UCL Research Ethics Committee to image the eye fundus of healthy volunteers with our prototype imaging system. Before to image the volunteers a drop of $1 \%$ tropicamide was administrated to the eye of the subject being tested.

Figure 12 shows a digitally refocus image of the optics disc of a healthy volunteer. The image produced 


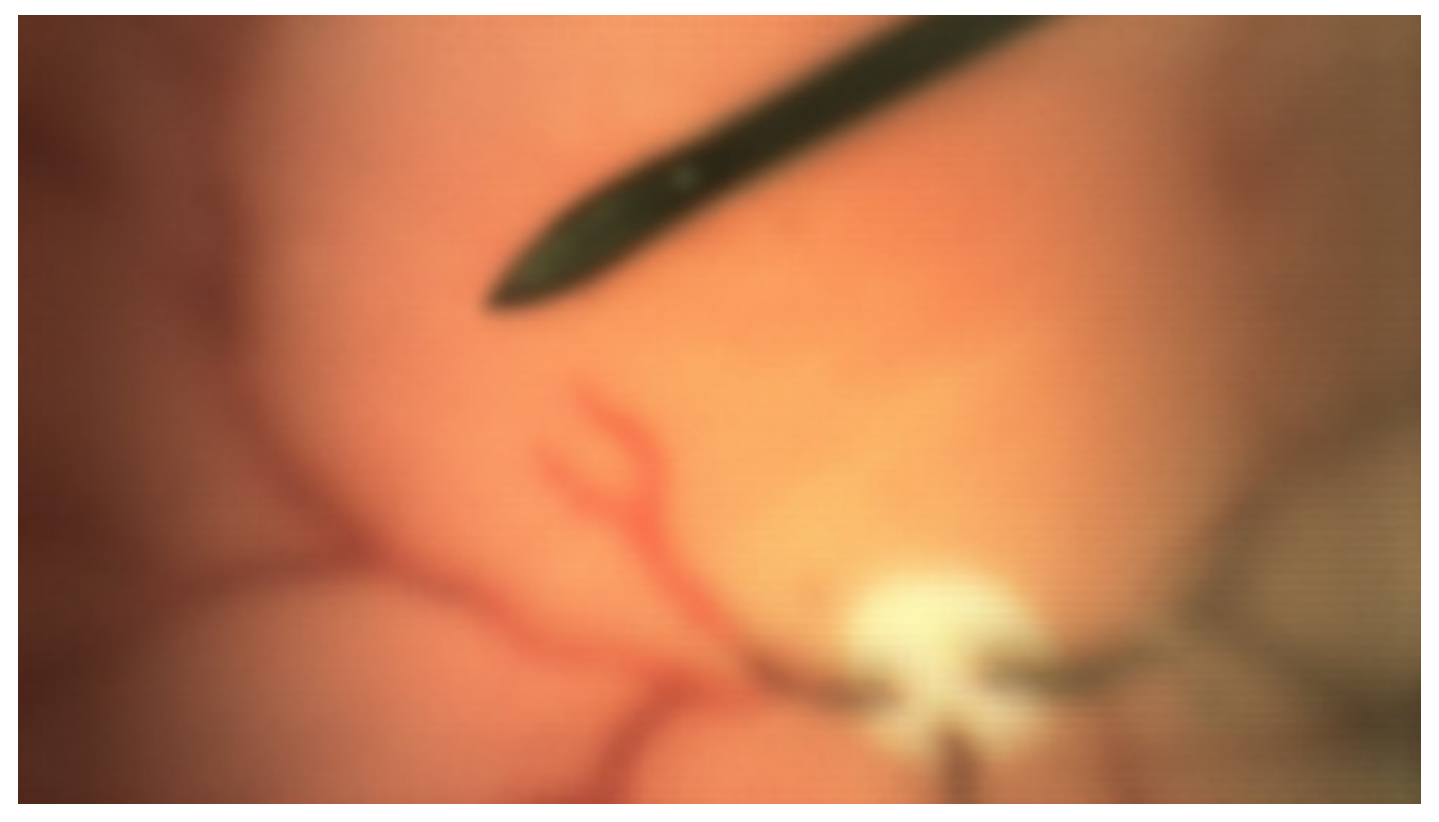

Figure 9. Digitally focused image on the needle using Raytrix RxLive software generated from fig. 7

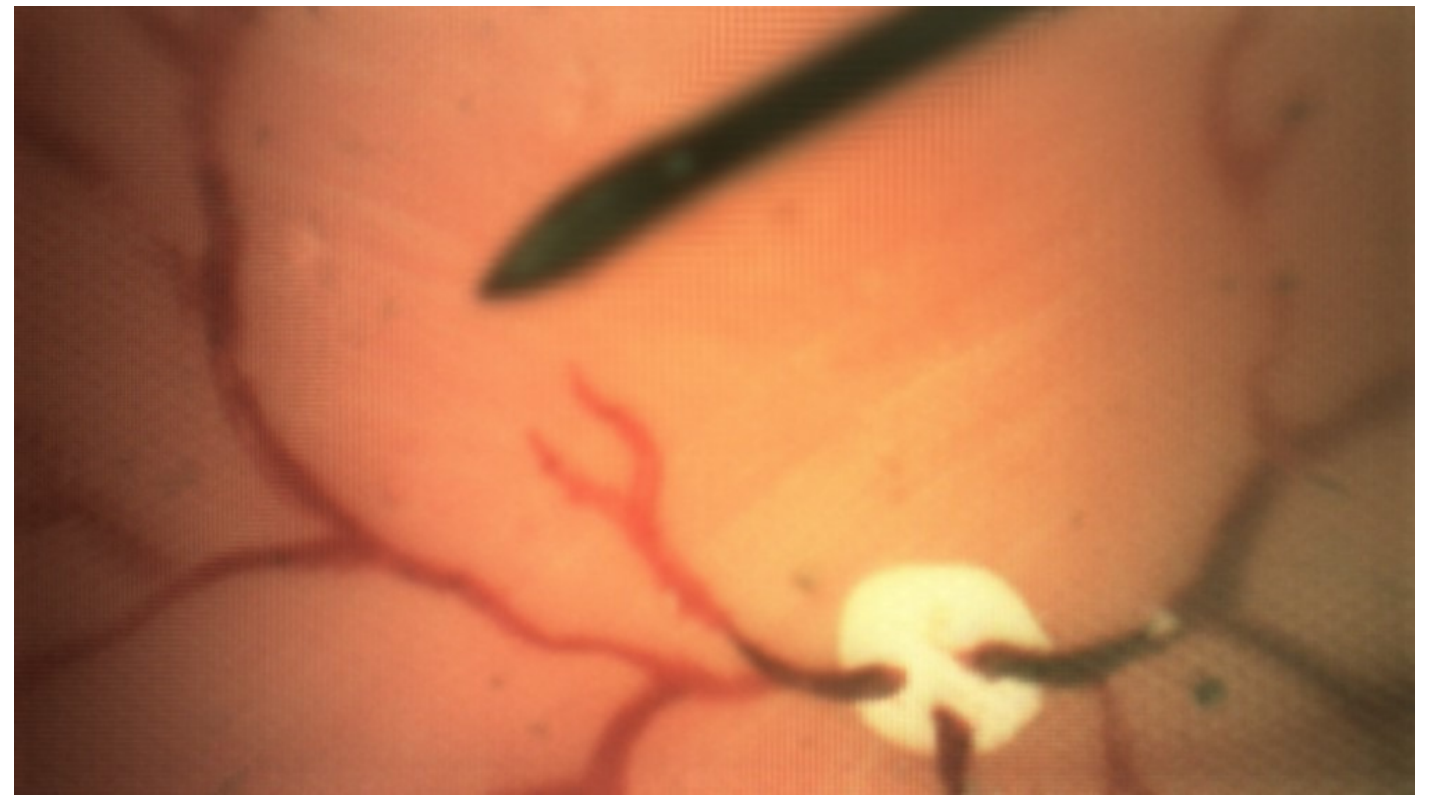

Figure 10. Total focus image using Raytrix RxLive Software generated from fig. 7. In this image both the retina and needle appear in focus.

has a very low contrast. Compared to the model eye the exposure time has to be increased by almost an order of magnitude. Therefore spurious back reflexion from the cornea and other optical elements become more of a problem. Hence when looking at a the plenoptic image use to generate the refocus image, one can see some bright spot in the top left corner of each individual micro-images (fig. 13). In addition the eye f-number of the subject is larger than the system designed f-number and some cross-talk appears as bright interstice between micro-images. Figure 12 was further process in order to enhance the optic disc and blood vessel. This is show in figure 14. The figure also exhibit some color artifacts. 


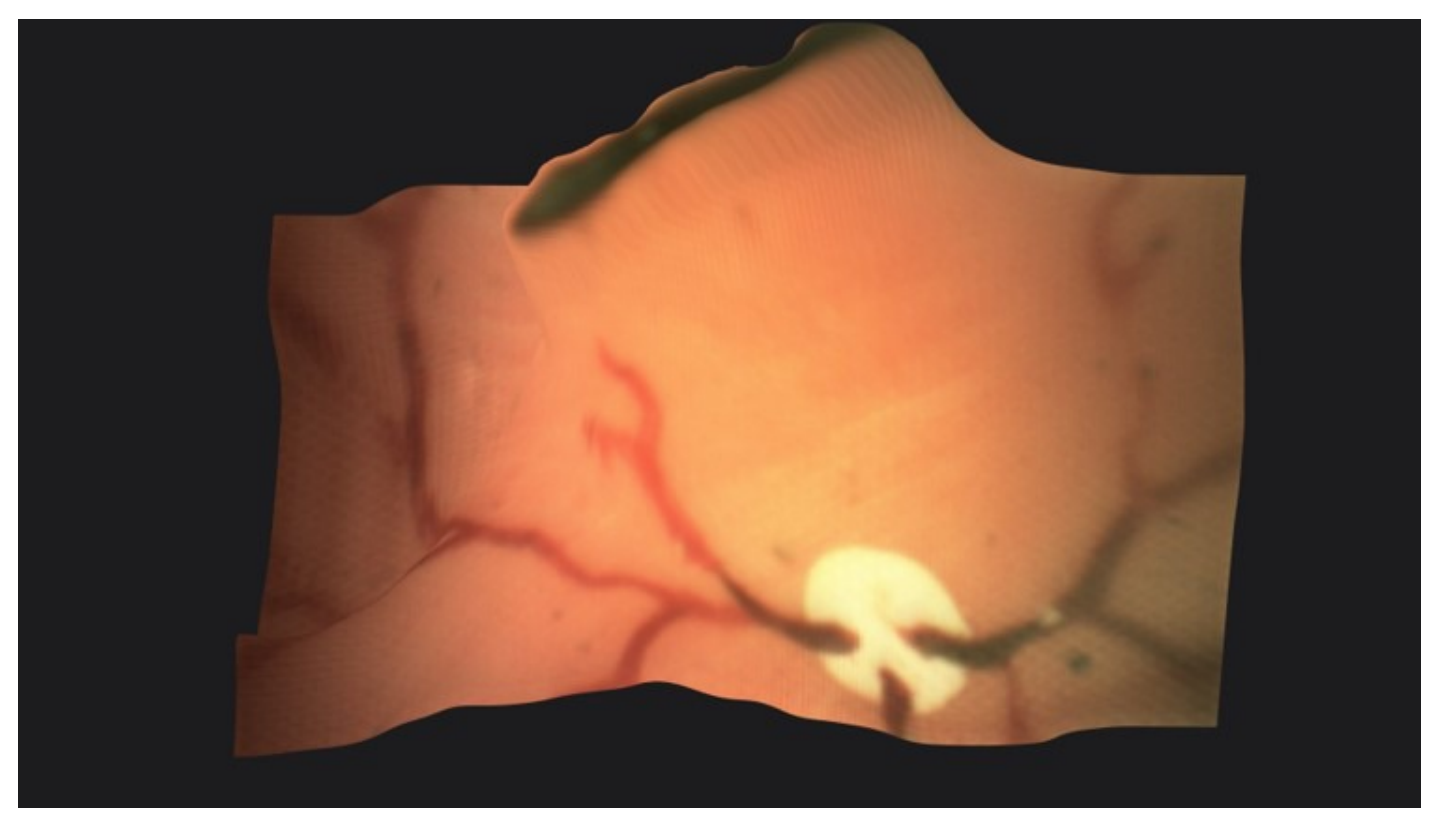

Figure 11. 3D map with the total focus texture apply on top of it using Raytrix RxLive Software generated from fig. 7. Some artifact are visible. The software requires the presence of sharp features to estimate their depth, in area with low contrast the depth has to be extrapolated. If the image is noisy and/or has a low resolution the algorithm cannot accurately estimate the depth.

Figure 12. Digitally focus In Vivo image of the optical disk of the left eye of a healthy volunteer. The image has a low contrast due to specular reflexion.

\section{DISCUSSION}

One well known limitation of the plenoptic camera is the relatively low available resolution. The focused plenoptic opththalmoscope implemented in this letter has a theoretical resolution at the retinal plane of about $60 \mathrm{lp} / \mathrm{mm}$ for a virtual depth equal to 4 and this resolution quickly drops for plane located closer to the eye lens (figure 4 ). While these planes located at longer virtual depth have lower spatial resolution, they on the contrary have higher 


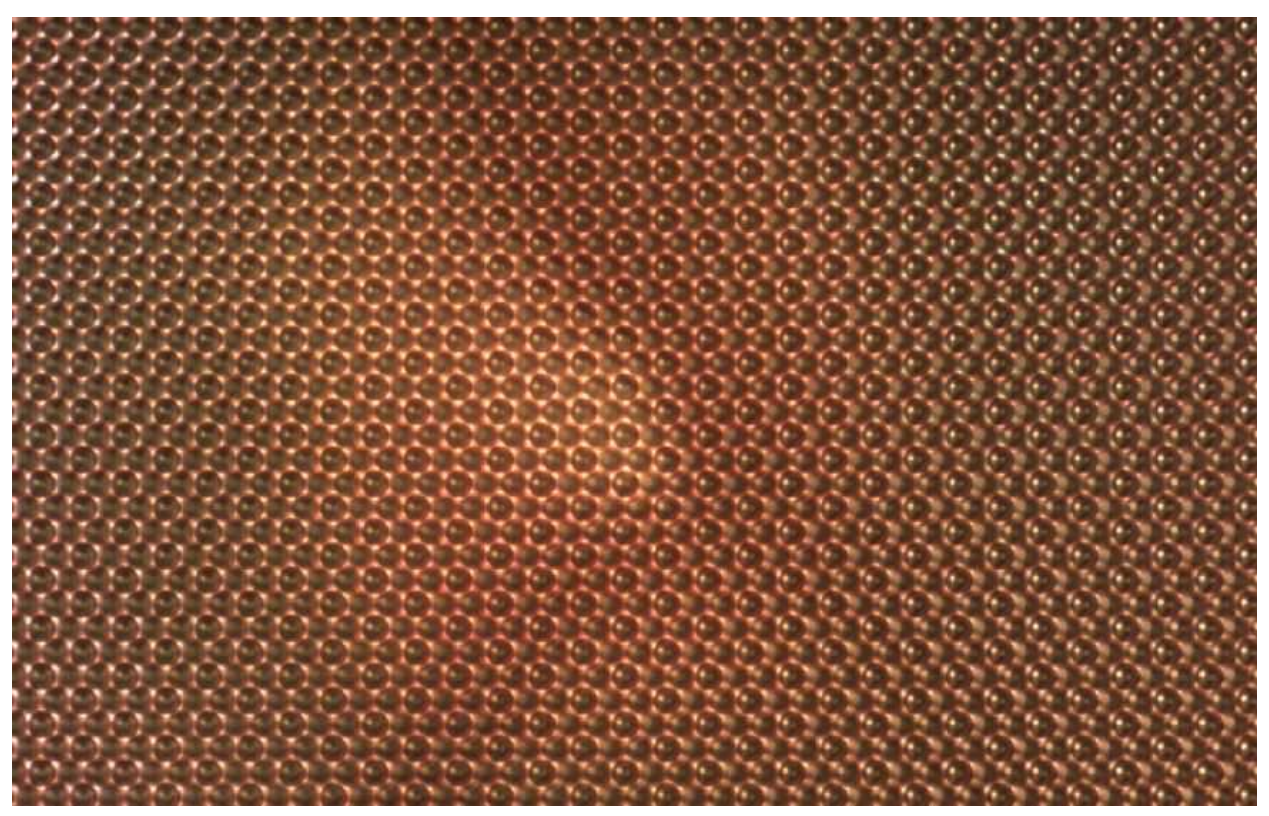

Figure 13. Cropped lightfield image from which Fig. 12 was generated. A small specular reflexion can be observed in the top left of each micro-images. Significant amount of Cross-talk visible as bright interstices between micro-images.

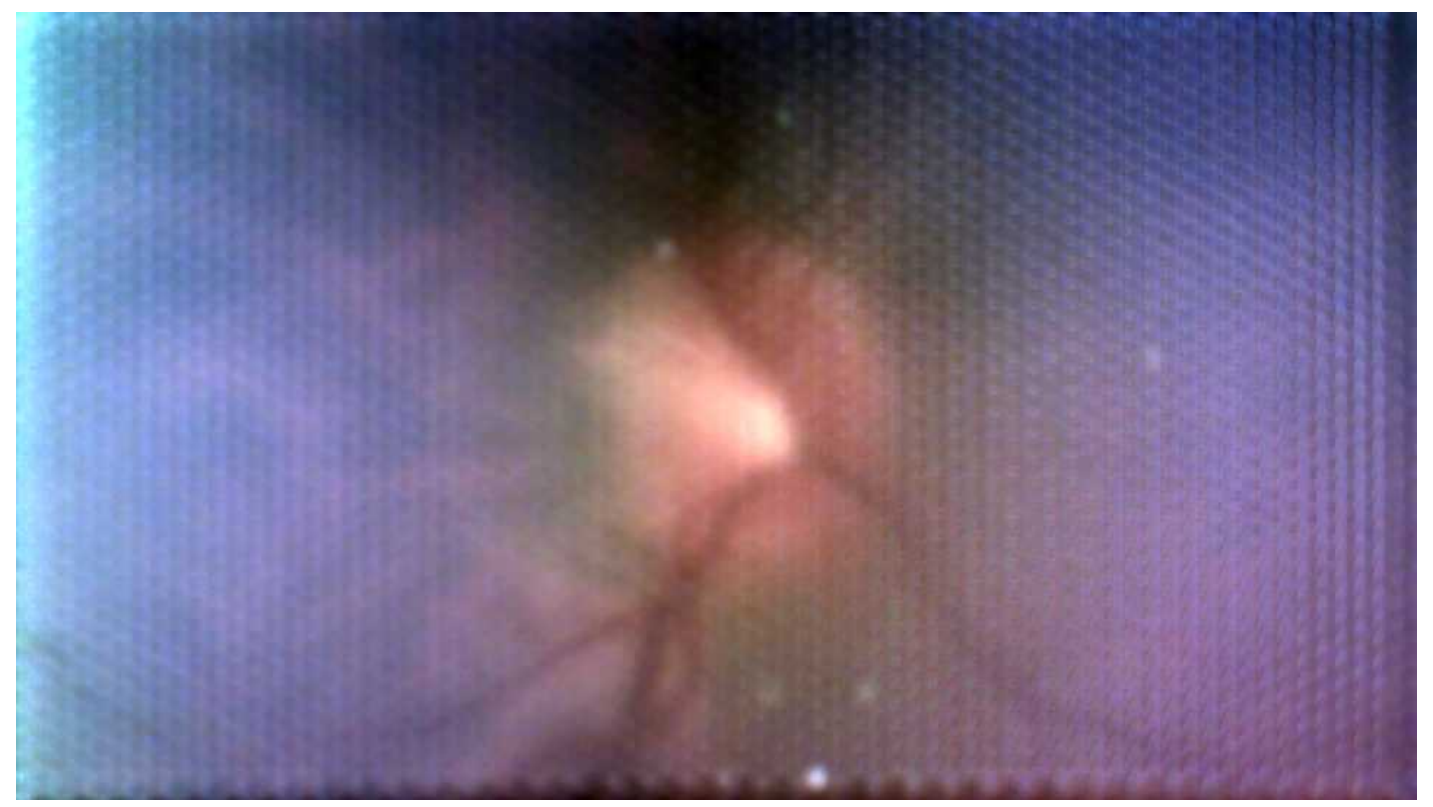

Figure 14. Post-processed Fig. 12. Colors, Constrast and brightness were ajusted to enhance the optical disck and blood vessel.

angular resolution (e.g. features present in these planes are imaged by more micro-lenses than for the planes at shorter virtual depth). More research needs to be conducted to establish the optimum sampling of the plenoptic function, which is also an important research topic in computational imaging. ${ }^{22}$

It is possible through the postprocessing of the disparities between the micro-images to compensate this reduction of spatial resolution. ${ }^{23,24}$ However this raise the question of what would be the optimal design parameters for a light field sensor in terms of virtual depth range, microlens array parameters and optical configuration. A plenoptic light transport framework ${ }^{25}$ has been proposed to guide the design of such system and to be able to 
compare different configuration. More recently, the equivalent camera array model ${ }^{26}$ was introduced.

In this proceeding, we did not investigate the post-processing and rendering of the fundus images in details. We have solely used the post-processing provided by RxLive, the software provided by Raytrix, the plenoptic sensor manufacturer. There are alternative and recent development in the field for computing the depth maps and rendering the refocus image and correct for distortion. ${ }^{27,28}$ Similarly we did not investigate how to postprocess the images in order to remove the corneal reflex. ${ }^{29}$ Prior to investigate this type of methods, we intend to improve the optical performances of our prototype. Especially we are designing a new system which further minimize stray light and specular reflexion.

In order to increase the focusing range of our system we would have to reduce the magnification of the main lens, at the same time this will reduce the field of view available for a given sensor size and require a short focal length lens. For a fixed eye pupil diameter, this will imply to reduce the micro-lenses f-number as defined by equation 1. We are investigating how to optimize the design of the focused plenoptic ophthalmoscope.

\section{ACKNOWLEDGMENTS}

We thank Peter West (UCL Institute of Ophthalmology) for providing necessary equipment. We acknowledge the support of EPSRC [EP/L016478/1], Fight for Sight [1728/29], Academy of Medical Sciences [SBF001/1002], European Research Council [714562], and Wellcome Trust [203145Z/16/Z].

\section{REFERENCES}

[1] Eckardt, C. and Paulo, E. B., "Heads-up surgery for vitreoretinal procedures: an experimental and clinical study," Retina 36(1), 137-147 (2016).

[2] Mohamed, Y. H., Uematsu, M., Inoue, D., and Kitaoka, T., "First experience of nDASEK with heads-up surgery: A case report," Medicine 96, e6906 (May 2017).

[3] Carrasco-Zevallos, O. M., Keller, B., Viehland, C., Shen, L., Waterman, G., Todorich, B., Shieh, C., Hahn, P., Farsiu, S., Kuo, A. N., Toth, C. A., and Izatt, J. A., "Live volumetric (4d) visualization and guidance of in vivo human ophthalmic surgery with intraoperative optical coherence tomography," Scientific Reports 6 (Oct. 2016).

[4] Abt, A., N., "Ophthalmic surgery using light-field microscopy," (Oct. 2015).

[5] Turola, M., Investigation of plenoptic imaging systems: a wave optics approach, $\mathrm{PhD}$ thesis, City University London (2016).

[6] Tunnermann, A., Gebhardt, S., and Fouckhardt, H., "Plenoptic Cameras," in [Tunable Micro-optics], Zappe, H. and Duppe, C., eds., 417-438, Cambridge University Press, Cambridge (2016). DOI: 10.1017/CBO9781139506052.017.

[7] Ihrke, I., Restrepo, J., and Mignard-Debise, L., "Principles of Light Field Imaging: Briefly revisiting 25 years of research," IEEE Signal Processing Magazine 33, 59-69 (Sept. 2016).

[8] Lam, E. Y., "Computational photography with plenoptic camera and light field capture: tutorial," Journal of the Optical Society of America A 32, 2021 (Nov. 2015).

[9] Tumlinson, R., A. and Everett, J., M., "Light Field Camera for Fundus Photography," (July 2011).

[10] Bedard, N., Meng, L., Namboodiri, V., Venkatesha, K. P. A., and Berkner, K., "Simultaneous capture of filtered images of the eye," (Nov. 2014).

[11] Lawson, M. E. and Raskar, R., "Methods and apparatus for retinal imaging," (Mar. 2014).

[12] Adam, M. K., Aenchbacher, W., Kurzweg, T., and Hsu, J., "Plenoptic Ophthalmoscopy: A Novel Imaging Technique," Ophthalmic Surgery, Lasers and Imaging Retina 47, 1038-1043 (Nov. 2016).

[13] Chen, H., Woodward, M. A., Burke, D. T., Jeganathan, V. S. E., Demirci, H., and Sick, V., "Human iris three-dimensional imaging at micron resolution by a micro-plenoptic camera," Biomedical Optics Express 8, 4514 (Oct. 2017).

[14] DeHoog, E. A., Novel fundus camera design, PhD, University of Arizona (2009).

[15] Watson, A. B., "Computing human optical point spread functions," Journal of Vision 15, 26-26 (Feb. $2015)$. 
[16] Perwass, C. and Wietzke, L., "Single lens 3d-camera with extended depth-of-field," in [ISEST/SPIE Electronic Imaging], 829108-829108, International Society for Optics and Photonics (2012).

[17] Georgiev, T. and Lumsdaine, A., "The multifocus plenoptic camera," 829908 (Jan. 2012).

[18] "Ophthalmic instruments - Fundamental requirements and test methods - Part2: Light hazard protection," British Standard BS EN ISO 15004-2:2007.

[19] Chen, X., Viehland, C., Carrasco-Zevallos, O. M., Keller, B., Vajzovic, L., Izatt, J. A., and Toth, C. A., "Microscope-Integrated Optical Coherence Tomography Angiography in the Operating Room in Young Children With Retinal Vascular Disease," JAMA Ophthalmology 135, 483 (May 2017).

[20] Read, S. P. and Fortun, J. A., "Visualization of the retina and vitreous during vitreoretinal surgery: new technologies," Current Opinion in Ophthalmology 28, 238-241 (May 2017).

[21] de Oliveira, A. O., de Matos, L., and Castro Neto, J. C., "Evaluation of retinal illumination in coaxial fundus camera," 994710 (Sept. 2016).

[22] Zhu, C., Yu, L., Yan, Z., and Xiang, S., "Frequency Estimation of the Plenoptic Function Using the Autocorrelation Theorem," IEEE Transactions on Computational Imaging 3, 966-981 (Dec. 2017).

[23] Georgiev, T., Chunev, G., and Lumsdaine, A., "Superresolution with the focused plenoptic camera," 78730X (Feb. 2011).

[24] Berkner, K., Toi, I., Wu, W., Meng, L., Bedard, N., and Balram, N., "Design Principles for Light Field Image Capture and Display," in [SID Symposium Digest of Technical Papers], 46, 342-345, Wiley Online Library (2015).

[25] Liang, C.-K. and Ramamoorthi, R., "A Light Transport Framework for Lenslet Light Field Cameras," ACM Transactions on Graphics 34, 1-19 (Mar. 2015).

[26] Mignard-Debise, L., Restrepo, J., and Ihrke, I., "A Unifying First-Order Model for Light-Field Cameras: The Equivalent Camera Array," IEEE Transactions on Computational Imaging 3, 798-810 (Dec. 2017).

[27] Zeller, N., Quint, F., Stterlin, M., and Stilla, U., "Investigating mathematical models for focused plenoptic cameras," in [Electronics and Telecommunications (ISETC), 2016 12th IEEE International Symposium on], 301-304, IEEE (2016).

[28] Hog, M., Sabater, N., Vandame, B., and Drazic, V., "An Image Rendering Pipeline for Focused Plenoptic Cameras," IEEE Transactions on Computational Imaging 3, 811-821 (Dec. 2017).

[29] Raskar, R., Agrawal, A., Wilson, C. A., and Veeraraghavan, A., "Glare aware photography: 4d ray sampling for reducing glare effects of camera lenses," in [ACM Transactions on Graphics (TOG)], 27, 56, ACM (2008). 